\title{
Data Association through Fusion of Target Track and Identification Sets
}

\author{
Erik Blasch \\ AFRL/SNAS \\ 2241 Avionics Cir. \\ WPAFB, OH 45433 \\ [erik.blasch@wpafb.af.mil]
}

\begin{abstract}
A joint probability data association tracking algorithm typically associates only position measurements[1]. With multiple-interacting targets in the presence of clutter, data association can be confused by spurious measurements. In this paper, we propose a set-based track and identification data association (SBDA) technique to leverage object identification information. We investigate the SBDA technique for a scenario in which a tracker has access to both coarse position measurements and belief identification information to enhance data association.
\end{abstract}

\section{Introduction}

The problem of multitarget tracking and identification (ID) is a subset of sensor fusion, which includes filtering, estimation, and prediction. One of the prominent tracking algorithms is the Joint Probability Data Association Filter (JPDAF) [1]. The JPDAF algorithm seeks to track a set of objects from only positional information, but improvements are underway to use a signal-detection analysis to track a object based on the highest signal return [2]. One way to enhance data association is to use target attributes to mitigate clutter [3]. To further enhance the capabilities of the JPDAF algorithm, it would be useful for a sensor to not only get the position of the target, but also the target identity. However, a true identity might not be known, so believable measurements must be used. A combined track and ID algorithm can improve track quality, mitigate clutter confusion, and enhance target recognition.

Kinematic and ID measurements can detect, track, and classify targets of interest. The ultimate objective of the tracker includes identifying targets as they move through space. In a dynamic and uncertain environment, the tracker must associate the correct target to the position measurements. Multitarget tracking in the presence of clutter has been investigated through the use of data association algorithms [1]. Likewise, other multisensor multiplatform fusion algorithms focus on identifying targets from multiple look sequences of sensor data [4].

\author{
Lang Hong \\ Dept. of Electrical Engineering \\ Wright State University \\ Dayton, OH 45435 \\ [lhong@cs.wright.edu]
}

The merging of these algorithms can be accomplished by investigating the mathematics of the algorithms. Track fusion uses kinematic measurements and ID fusion uses target-feature measurements to update state matrices. By utilizing the merits of data association in multilevel data association, we seek to simultaneously track and identify targets [5].

In tracking approaches that use data association, there is an assumption that the information for tracking is provided through position measurements. The problem is that the tracker must isolate the target of interest from the cluttered position measurements. If position measurement information is dense, the tracker can make an incorrect assignment of the position measurements to target tracks. As an example, Figure 1 below shows a case in which the position measurements cause the tracker to get confused. In this case, object 1's and object 2's position measurements fall within the kinematic gates of both objects.

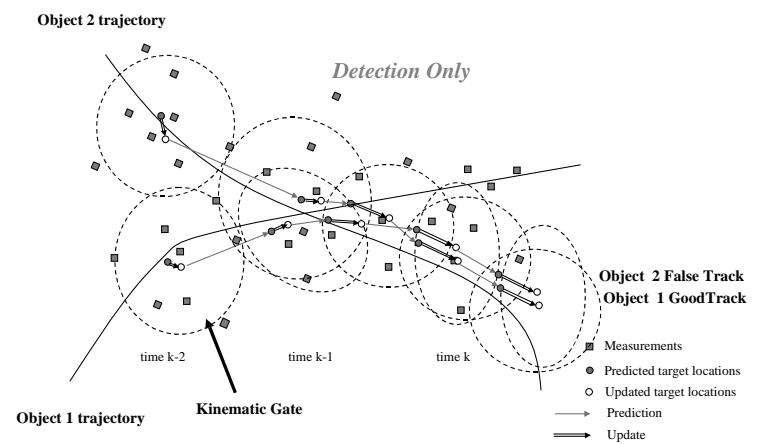

Figure 1. Data Association Problem with only Position Measurements.

As we can see from the Figure 1 (far left), a kinematic gate can isolate position measurements that are near the predicted measurement for each object's track. In the case that one of the true measurements falls within the kinematic gate of the predicted position, that measurement would be designated as the true position measurement. If position measurements from another object fall within the predicted kinematic gate of the object track (Figure 1 
E. Blasch and L. Hong, “Data Association through Fusion of Target track and Identification Sets,” Fusion 2000, Paris, France. July 1013, 2000, TuD2_17-TuD2_23

middle), the position measurement of object 2 would be assigned to object 1's track which would be an incorrect assignment. Once the tracker locks on to another object, or uses the position hits of the other object's clutter, the tracker assumes that the hits of the second object are true hits for the first object (Figure 1 right).

One way to correct for this measurement-to-target assignment mistake is to leverage other information, such as the target identity to help resolve which position measurements are assigned to specific object tracks. For example, we could use a high range resolution (HRR) sensor to ID an moving target and use the false and true HRR scans as positional information [5]. To illustrate how the ID information may help in data association, Figure 2 illustrates the process of how a target-ID can refine the positional measurement to select the validated measurement from the cluttered measurements.

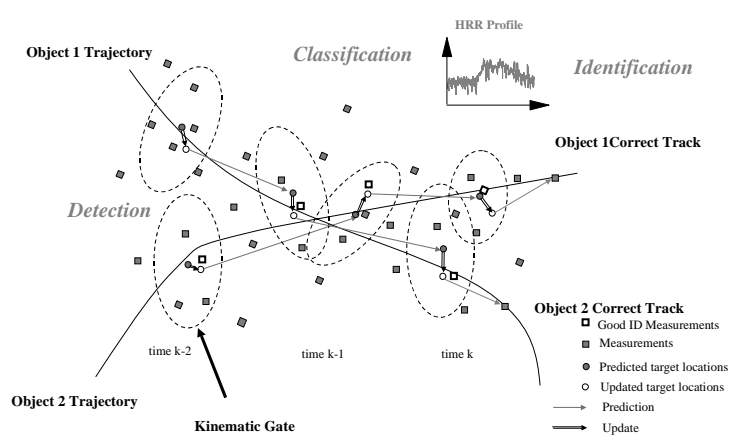

Figure 2. Data Association using ID and Position Measurements.

A few tracking and identification algorithms have been proposed $[6,7,8]$. These approaches combine target recognition and classification techniques with tracking. Layne [9] utilizes an automatic target recognition (ATR) and tracking filter in a multiple model estimator (MME) approach. We seek to expand on this idea by allowing for the capability to ID relevant targets. Identification goes beyond recognition by assigning a single target ID to each target. By incorporating a general theory for target identity, any combination of sensors and targets can be tracked for a variety of applications such as medical, manufacturing, and economic scenarios.

This paper develops a set-based track and identification data association (SBDA) technique to distinguish between multiple moving targets in clutter. Section 2 overviews track and ID data association fusion. Section 3 describes the problem formulation and Section 4 details mathematics of the algorithm. Section 5 presents results and Section 6 draws some conclusions.

\section{Track and ID Fusion}

The ability to perform track and ID fusion requires sensor-processed classifications from different levels.
These levels could be generic(car), feature(wheeled), type(sedan) or specific(license plate). Like multitarget data association algorithms for accurately tracking targets in the presence of clutter, we assume that detected targets can be tracked from a sequence of center-of-gravity and pose positional data. However, for a given sensor/target scenario, we assume detected classifications can effectively discern target identity. Identity information can be achieved either through experience of target movement, training, or predicted. For example, identifying a target requires the correct estimate of the target's orientation and speed. Two targets of the same type may be crossing in space, but since they can not occupy the same location, they would each have a different orientation relative to the sensor. By exploiting the orientation and speed information, each target can be assessed for the correct track-ID association.

The ability of an sensor to track and ID targets simultaneously includes finding the target center for tracking, determining the target pose, and searching the neighboring features for discerning salient features to associate the features to a specific class of targets. By partitioning kinematic and ID sensor data, associations at different levels can be used for either coarse(track) or fine(ID) target analysis. For example, features[5] can be used to get an identity with uncertainty; however if many features are fused, the identity increases and helps to eliminate clutter. Identifying a target is a subset of ATR in which the tracker must use the available features to discern the object. Certain features are inherently more useful in recognizing a target than others. For instance, identifying a large car versus a small car would result from an analysis of the length-to-width ratio. Additionally, decoupling information can be used for a single-platform observer to fuse information from a sequence of sensor data or for a multiple-platform scenario [4] in which fusing is performed from different geometrical positions. For further information on the development of the belief-ID derivation, see [5].

The problem of track level and ID-level fusion has characteristic tradeoffs about which the tracker must decide. For close targets, it is useful to keep an accurate track on multiple targets. The intelligent processor performs target-to-ID association at multiple levels and can either track targets at a low resolution or ID targets at a higher resolution. By leveraging knowledge about target types, fusion algorithms can significantly reduce processing time for tracking and identifying targets. For separated targets, resources may exist to identify each target. Hence, due to a limited set of resources and/or processor time, a trade-off exists between the identification and tracking of a target.

\section{Problem Formulation}

Consider an environment in which a tracker is monitoring 
E. Blasch and L. Hong, “Data Association through Fusion of Target track and Identification Sets,” Fusion 2000, Paris, France. July 1013, 2000, TuD2_17 -TuD2_23

multiple moving targets with stationary clutter. By assumption, the tracking sensor is able to detect target signatures. Assume that the 2-D region is composed of $T$ targets with $f$ features. Dynamic target measurements $z$ are taken at time steps $k$, which include target kinematic and identification features $\mathbf{z}(\mathrm{k})=\left[x_{t}(\mathrm{k}), \mathrm{f}_{1}, \ldots \mathrm{f}_{\mathrm{n}}\right]$.

Any sensor can measure independently of the others, and the outcome of each measurement may contain kinematic or feature variables indicating any target. The features for each sensor are similar, but need to be extracted and applied to the separate targets for classification. The probability density of each measurement depends on whether the target is actually present or not. Further assume that a fixed number of kinematic and feature measurements will be taken at each time interval, where we model the clutter composing spurious measurements. A final decision form the SBDA algorithm is rendered as to which $[x, y]$ measurement is associated with the targettype.

The multisensor-multitarget tracking and identification problem is to determine which measured kinematic features should be associated with which ID features in order to optimize the probability that targets are tracked and identified correctly after $z$ measurements. The multilevel feature fusion problem is formulated and solved by using concepts developed using the belief filter [5]. For the symmetric-target case, the "association rule" associates the measurement with the highest target probability.

\section{Track and ID Data Association}

\subsection{Tracking Belief Filter}

The target state and true measurement are assumed to evolve in time according to:

$$
\begin{aligned}
& \mathbf{x}(k+1)=\mathbf{F}(k) \mathbf{x}(k)+\mathbf{v}(k) \\
& \mathbf{z}(k)=\mathbf{H}(k) \mathbf{x}(k)+\mathrm{w}(k)
\end{aligned}
$$

where $\mathbf{v}(k)$ and $\mathbf{w}(k)$ are zero-mean mutually independent white Gaussian noise sequences with known covariance matrices $\mathbf{Q}(k)$ and $\mathbf{R}(k)$, respectively. We assume each target has a separate track and set up multiple state equations. Spurious measurements are uniformly distributed in the measurement space. Tracks are assumed initialized at an initial state estimate $\mathbf{x}(0)$, contain a known number of targets determined from the scenario, and have associated covariances [1].

The tracking ID filter devotes equal attention to every validated kinematic or ID measurement and cycles through object measurements until a believable set of object IDs is refined to associate one object per track. For an initial set of measurements, a hypothesized number of tracks and objects of interest is assumed to comprise the entire set. Objects are possible position measurements without ID confirmation. Successive measurements and updates from the combined feature and track measurements determine the set of plausible targets. The measurement filter assumes the past is summarized by an approximate sufficient statistic - track state and ID state estimates (approximate conditional mean) and covariances for each object.

The belief measurement information $\mathbf{B e l}_{\mathrm{k}}^{t}=\mathbf{M} \bullet \mathbf{B e l}_{\mathrm{k}-1}^{t}$, derived from the classification measurements of the target profile, represents the belief update states of the ID measurements. The $\mathbf{M}$ matrix is the Markov transition matrix, which represents the similarity of objects. The similarity of objects represents how the belief in an object type may be related to other objects of the same or different type.

The measurement-to-track association probabilities are computed across the objects and these probabilities are computed only for the latest set of measurements. The conditional probabilities of the joint track-ID association events pertaining to the current time $k$ are defined as $\theta_{j o t_{k}}$, where $\theta_{j o t_{k}}$ is the event that object center-of-gravity measurement $j$ originated from object $o$ and track $t, j=1$, $m_{\mathrm{k}} ; o=0,1, \ldots, \mathrm{O}_{\mathrm{n}}$, where $m_{\mathrm{k}}$ is the total number of measurements for each time step and $\mathrm{O}_{n}$ is the unknown number of objects. Note, for purposes of tracking and ID, we define $i=1, \ldots, m_{\mathrm{k}}$ for the entire measurement set while $j=1, \ldots, m_{\mathrm{k}}$ is for tracking and $o=1 \ldots, m_{\mathrm{k}}$ is for object ID.

A validation gate for each object bounds the believable joint measurement events, but not in the evaluation of their probabilities. The plausible validation matrix: $\Omega=$ $\left|\omega_{j \mathrm{t}}\right|$ is generated for each object of a given track which comprises binary elements that indicate if measurement $j$ lies in the validation gate of track $t$. The index $t=0$ represents for "the empty set of tracks" and the corresponding column of $\boldsymbol{\Omega}$ includes all measurements, since each measurement could have originated from clutter, false alarm, or the true object [1].

For a track event, we have:

$$
\left|\hat{\omega}_{j t}(\theta)\right| \Delta\left\{\begin{array}{cc}
1 & \text { if } \theta_{j \mathrm{t}}^{\mathrm{i}} \in \theta ;[\mathbf{z}]_{\mathrm{k}}^{\mathrm{i}} \subset t \\
0 & \text { otherwise }
\end{array}\right.
$$

where measurement $[\mathbf{z}]_{\mathrm{k}}^{\mathrm{i}}$ originated from track $t$

For an ID-belief event, which is above a predetermined ID threshold,

$$
\left|\hat{\omega}_{o \mathrm{O}}(\theta)\right| \Delta\left\{\begin{array}{cc}
1 & \text { if } \theta_{o \mathrm{O}}^{\mathrm{i}} \in \theta ;[\mathbf{B e l}]_{\mathrm{O}_{\mathrm{k}}}^{\mathrm{i}} \Leftrightarrow o \\
0 & \text { otherwise }
\end{array}\right.
$$

where measurement $[\mathbf{B e l}]_{\mathrm{O}_{\mathrm{k}}}^{\mathrm{i}}$ is associated $(\Leftrightarrow)$ with object o. 
E. Blasch and L. Hong, "Data Association through Fusion of Target track and Identification Sets,” Fusion 20@e, Paris, Frange. 9uby 10113, 2000, TuD2_17 -TuD2_23

Since SBDA is tracking multiple objects, $o$, assuming one for each track, $t$, SBDA has to determine the ID-belief in each object from a known database comparison. While these IDs are processed over time to discern the object, for each measurement, SBDA must determine if the trackID measurements are plausible. SBDA uses the current ID-beliefs to update the association matrix. If the belief in the object is above a threshold, SBDA declares the measurement $i$, to be plausible for the target. Note, for plausibility, the threshold is lower than an ID declaration.

\subsection{Data Association}

Since we have assessed the continuous-kinematic information and the discrete-classification event, we can now assess the intersection of kinematic and ID information for simultaneous object tracking and ID. Note, ID goes beyond object detection, recognition, and classification, where we define ID as the classification of an object-type for a given track to associate an object classification to a track. For instance, two objects of the same class still need to be associated with a specific track. We need to address feasible events for either a validated kinematic measurement or a validated ID. A kinematic-ID joint association event consists of the values in $\Omega$ corresponding to the associations in $\theta_{\text {jot }}$,

$$
\left|\hat{\omega}_{\text {jot }}(\theta)\right| \Delta\left\{\begin{array}{cc}
1 & \text { if } \theta_{j \mathrm{ot}}^{i} \in \theta * \\
0 & \text { otherwise }
\end{array}\right.
$$

where $(*)$ measurement $[\mathbf{z}]_{\mathrm{k}}^{\mathrm{i}}$ originated from track $t$ with a $[\text { Bel }]_{\mathrm{o}_{\mathrm{k}}}^{\mathrm{i}}$ for a given $O_{\mathrm{ot}}$ and

$$
\hat{\omega}_{j o t}(\theta)=\hat{\omega}_{j \mathrm{t}}(\theta) \oplus \hat{\omega}_{o O}(\theta)
$$

Note, we define the indices as jot since $O$ is the number of objects which is equal to the number of tracks.

These joint events will be assessed with “ $\beta$ ” weights [1] to determine the extent of belief in the associations. To process the believability of track associations, augmented with the ID information, we set up a matrix formulation. For example, we have a set of kinematic measurements $\mathbf{z}_{i}$ with a $\mathbf{B e l}_{O}$ and put them into the event association matrix as illustrated in Figure 3. The upper left of a box represents the track information where a " 1 " indicates the kinematic measurement lies within a gated position measurement. The lower right represents the belief in an object type of any class except the unknown class where a believable object receives a " 1 ". Columns are for tracks and rows for measurements. These generalized equations propagate ID-filtered, predicted ID measurements in time.

In the case of joint association, SBDA processes event matrices with an "AND" function allowing for plausible events from either the track or classification plausible events. To determine the event plausibility, SBDA uses
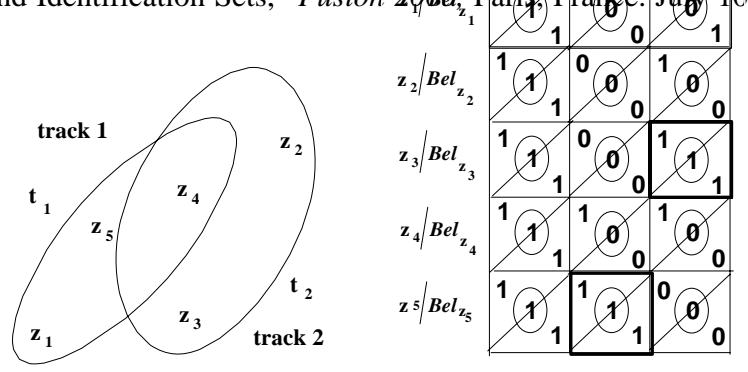

Figure 3. Tracking and Classification Joint Association

the validation region for track measurements and uses a threshold, or classification gate, to determine the match for a target-type ID associated with a given track. Figure 4 illustrates the "AND” function. Note, SBDA rejects non-believable measurements and measurements that lie outside the kinematic validation gate.

For the determination of the weights assigned to these associations, SBDA needs to set up the state and probability values. A track-ID association event has [1]

i) a single object-type measurement from a source:

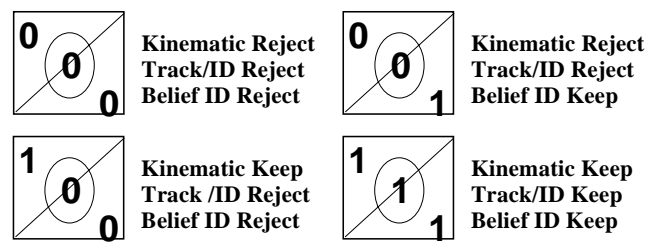

Figure 4. Believable Events for the association matrix.

$$
\sum_{o=0}^{\mathrm{O}_{\mathrm{n}}} \hat{\omega}_{j o t}\left(\theta_{j \mathrm{ot}}^{i}\right)=1 \quad \forall j
$$

ii) and at most one object-type measurement ID originating from a object for a given track:

$$
\delta_{t}(\theta) \stackrel{\Delta}{=} \sum_{j=1}^{m_{\mathrm{k}}} \hat{\omega}_{j o t}\left(\theta_{j \mathrm{ot}}^{i}\right) \leq 1
$$

The generation of event matrices, $\Omega$ for each track, corresponding to ID events can be done by scanning $\boldsymbol{\Omega}$ and picking one unit/row and one unit/column for the estimated set of tracks except for $t=0$. In the case that SBDA has generated event matrices for an estimated number of tracks with different object types, SBDA needs to assess the combination of feature measurements to infer the correct number of tracked objects that comprise the set. The binary variable $\delta_{t}\left(\theta_{\text {jot }_{\mathrm{k}}}\right)$ is called the track detection indicator [1] since it indicates whether a measurement is associated with the object $o$ and track $t$ in event $\boldsymbol{\theta}_{\text {jot }}$, i.e. whether it has been detected. 
E. Blasch and L. Hong, “Data Association through Fusion of Target track and Identification Sets,” Fusion 2000, Paris, France. July 1013, 2000, TuD2_17 -TuD2_23

The measurement association indicator

$$
\tau_{j}\left(\theta_{j o t_{k}}\right) \stackrel{\Delta}{=} \sum_{j=1}^{m_{k}} \hat{\omega}_{j o t}\left(\theta_{j o t_{k}}\right)
$$

indicates measurement $j$ is associated with the track $t$ in event $\boldsymbol{\theta}_{\text {jot }}$.

The number of false measurements in event $\theta$ is

$$
\phi(\theta)=\sum_{j-1}^{m}\left[1-\tau_{j}(\theta)\right]
$$

The joint association event probabilities are, using Bayes' Formula:

$$
\begin{aligned}
\mathrm{P}\left\{\theta(k) \mid \mathbf{Z}^{k}\right\} & =\mathrm{P}\left\{\theta(k) \mid \mathbf{Z}(k), \mathrm{m}(k), \mathbf{Z}^{k-1}\right\} \\
& =\frac{1}{\mathrm{C}} \mathrm{P}\left[\mathbf{Z}(k) \mid \theta(k), \mathrm{m}(k), \mathbf{Z}^{k-1}\right] \mathrm{P}\{\theta(k) \mid \mathrm{m}(k)\} \\
& =\frac{1}{\mathrm{C}} \prod_{j=1}^{\mathrm{m}(k)-\phi(k)} \mathrm{V}\left\{\mathrm{f}_{t_{t}}(k)\left[\mathbf{z}_{j}(k)\right]\right\}
\end{aligned}
$$

where $c$ is the normalization constant.

The number of measurement-to-target assignment events $\theta(k)$ is the number of targets to which a measurement is assigned under the same detection event $[\mathrm{m}(k)-\phi]$. The target indicators $\delta_{\mathrm{t}}(\theta)$ are used to select the probabilities of detecting and not detecting events under consideration.

\subsection{Fused Track and ID State Estimation}

Assuming the targets conditioned on the past observations are mutually independent, the decoupled state estimation uses the marginal association probabilities, which are found from the joint probabilities by summing all the joint events in which the marginal track and classification events result. The beta weights [1] are:

$$
\begin{aligned}
\boldsymbol{\beta}_{j o_{\mathrm{k}}}^{t}= & \mathrm{P}\left\{\boldsymbol{\theta}_{j o t_{\mathrm{k}}} \mid \mathbf{Z}^{k}\right\} \\
& =\sum_{\theta} \mathrm{P}\left\{\theta_{j o t_{\mathrm{k}}} \mid \mathbf{Z}^{k}\right\} \hat{\omega}_{j o}\left(\theta_{j o t_{\mathrm{k}}}\right)
\end{aligned}
$$

SBDA decomposes the object-state estimation with respect to the location of each object of the latest set of validated belief-set and kinematic-set measurements. The measurements have been used to get the classification beliefs in the object types, to set up a simultaneous tracking and ID recursion for each object in the set, where ID is the classification of each object for a given track of data. For each object measurement, we use the total probability theorem to get the conditional mean of the state at time $k$ can be written as:

$$
\hat{\mathbf{X}}_{\mathrm{k} \mid \mathrm{k}}^{t}=\sum_{\mathrm{i}=0}^{m_{\mathrm{k}}^{o}} \hat{\mathbf{X}}_{\mathrm{k} \mid \mathrm{k}}^{t i} \boldsymbol{\beta}_{\mathrm{k}}^{t i}
$$

where $\hat{\mathbf{X}}_{\mathrm{k} \mid \mathrm{k}}^{t}$ is the updated state conditioned on the event that the $i^{\text {th }}$ validated object measurement is correct for track $t$. The covariance propagation is:

$$
\begin{aligned}
& \mathbf{P}_{\mathrm{k} \mid \mathrm{k}-1}^{t}=\mathbf{F}_{\mathrm{k}-1}^{t} \mathbf{P}_{\mathrm{k}-1}^{t}\left(\mathbf{F}_{\mathrm{k}-1}^{t}\right)^{\mathrm{T}}+\overline{\mathbf{Q}}_{\mathrm{k}-1}^{t}, \\
& \overline{\mathbf{Q}}_{\mathrm{k}}=\left[\begin{array}{cc}
\mathbf{Q}_{\mathrm{k}} & 0 \\
0 & \mathbf{B}_{\mathrm{k}}
\end{array}\right]
\end{aligned}
$$

where

for each track $t$.

We can obtain the innovation covariance $S_{k}$ with the associated $\mathbf{R}_{\mathrm{k}}$ and measured $\mathbf{D}_{\mathrm{k}}$ by:

$$
\mathbf{S}_{\mathrm{k}}^{t}=\mathbf{H}_{\mathrm{k}}^{o_{t}^{t}} \mathbf{P}_{\mathrm{k} \mid \mathrm{k}-1}^{t}\left(\mathbf{H}_{\mathrm{k}}^{o}\right)^{\mathrm{T}}+\overline{\mathbf{R}}_{\mathrm{k}}^{t} \text {, where } \overline{\mathbf{R}}_{\mathrm{k}}=\left[\begin{array}{cc}
\mathbf{R}_{\mathrm{k}} & 0 \\
0 & \mathbf{D}_{\mathrm{k}}
\end{array}\right]
$$

Since $\mathbf{S}_{\mathrm{k}}$ is the innovation covariance update, we can use $\mathrm{S}_{\mathrm{k}}$ to gate measurements based on the uncertainty with the associated track and IDs.

Validation: At $k$, two measurements are available for object $o$ for a given track $t: \mathbf{z}_{\mathrm{k}-1}^{\mathrm{T}}$, and $\mathbf{z}_{\mathrm{k}}^{\mathrm{T}}$, from which position, velocity, pose, and ID features can be extracted form the belief track vectors. Validation, based on track and ID information, is performed to determine which track-belief measurements fall into the kinematic region of interest. Validation can be described as

$$
\left(\mathbf{z}_{k}^{t}-\hat{\mathbf{z}}_{\mathrm{k} \mid \mathrm{k}-1}^{\mathrm{lt}}\right)^{\mathrm{T}}\left[\mathbf{S}_{\mathrm{k}}^{t}\right]^{-1}\left(\mathbf{z}_{\mathrm{k}}^{t}-\mathbf{z}_{\mathrm{k} \mid \mathrm{k}-1}^{l t}\right) \leq \gamma \quad \text { for } l=1 \ldots m_{\mathrm{k}}^{\mathrm{o}}
$$

where $\gamma$ is a validation threshold obtained from a $\chi^{2}$ table for a degree of freedom of 14 (4 for kinematic states and 10 for target beliefs) and $\mathbf{S}_{\mathrm{k}}$ stands for the largest among the predicted track belief covariance, i.e., $\operatorname{det}\left(\mathbf{S}_{\mathrm{k}}\right) \geq \operatorname{det}\left(\mathbf{S}_{\mathrm{k}}^{t}\right)$ for $t=1,2, \ldots, n$. where $n$ is the number of states $\hat{\mathbf{z}}_{\mathrm{k} \mid \mathrm{k}-1}$ is a combined predicted track belief given by $E\left\{\mathbf{z}_{k} \mid\left\{\underline{\beta}^{\varsigma}\right\}_{O=1}^{s}, Z^{\mathrm{k}-1}\right\}$ where $s$ is the set of object beliefs for a track.

Data association for $\beta_{I}^{t i}$ : Data association performed for each belief object-track is similar to that in PDA and the details can be found in [1]. The association probabilities for $l$ validated object measurements are:

$$
\begin{aligned}
& \boldsymbol{\beta}_{l}{ }^{t}= \frac{e_{l}^{t}}{m_{\mathrm{k}}^{o}}, l=1,2, \ldots, m_{\mathrm{k}}^{0} \\
& \boldsymbol{\beta}_{0}{ }^{t}= \frac{b}{m_{l=1}^{o} e_{l}^{t}}, \\
& b+\sum_{l=1}^{m_{\mathrm{k}}} e_{l}^{t}
\end{aligned}
$$


E. Blasch and L. Hong, "Data Association through Fusion of Target track and Identification Sets,” Fusion 2000, Paris, France. July 1013, 2000, TuD2_17 -TuD2_23

$$
\begin{aligned}
& \text { where } e_{l}^{t}=P_{\mathrm{G}}^{-1} N\left(\underline{0}, \mathrm{~s}_{\mathrm{k}}^{t}\right) \\
& b=m_{\mathrm{k}}^{\mathrm{o}}\left(1-P_{\mathrm{D}} P_{\mathrm{G}}\right)\left[P_{\mathrm{D}} P_{\mathrm{G}} V_{\mathrm{k}}\right]^{-1}
\end{aligned}
$$

with $m_{\mathrm{k}}^{\mathrm{o}}$ defining the number of validated object measurements, $P_{\mathrm{G}}$ assessing the probability that augmented belief track measurements fall into the validation region, and $P_{\mathrm{D}}$ representing a detection probability. The volume of the validation gate is

$$
V_{\mathrm{k}}=C_{\mathrm{d}} \gamma^{\mathrm{d} / 2}\left|\mathrm{~S}_{\mathrm{k}}\right|^{1 / 2},
$$

where $\mathbf{C}_{\mathrm{d}}$ is the volume of the unit hypersphere of dimension $d$, the dimension of the augmented belief-track measurement .

Kinematic belief-probabilistic update: The object beliefprobabilistic track update is performed as a full rate system to combine the state, innovation, and covariances.

$$
\hat{\mathbf{X}}_{k \mid \mathrm{k}}^{t}=\hat{\mathbf{X}}_{\mathrm{k}-1 \mid \mathrm{k}-1}^{t}+\mathbf{W}_{\mathrm{k}}^{t} \sum_{l=1}^{\mathrm{m}_{\mathrm{k}}^{\mathrm{k}}} \boldsymbol{\beta}_{l k}^{t} \boldsymbol{v}_{l k}^{t}
$$

and

$$
\begin{aligned}
& \mathbf{P}_{k \mid k}^{t}=\boldsymbol{\beta}_{0}^{t} \mathbf{P}_{k \mid k-1}^{t}+\left(1-\boldsymbol{\beta}_{0}^{t}\right) \mathbf{P}_{k \mid k}^{*}+ \\
& \mathbf{W}_{\mathrm{k}}{ }^{t}\left[\sum_{l=1}^{\mathrm{m}_{\mathrm{k}}^{\mathrm{o}}} \boldsymbol{\beta}_{l k}^{t} \boldsymbol{v}_{l k}^{t}\left[\boldsymbol{v}_{l k}^{t}\right]^{\mathrm{T}}-\boldsymbol{v}_{k}^{t}\left[\boldsymbol{v}_{k}{ }^{t}\right]^{\mathrm{T}}\right]\left(\mathbf{W}_{k}^{t}\right)^{\mathrm{T}}
\end{aligned}
$$

where

$$
\begin{aligned}
& \mathbf{P}_{k \mid k}^{*}=\left[\mathrm{I}-\mathbf{W}_{\mathrm{k}}^{t} \mathbf{H}_{\mathrm{k}}^{o}\right] \mathbf{P}_{\mathrm{k} \mid \mathrm{k}-1}^{t} \text { and } \boldsymbol{v}_{k}=\sum_{l=1}^{m_{\mathrm{k}}^{o}} \boldsymbol{\beta}_{l \mathrm{k}}^{t} \boldsymbol{v}_{l \mathrm{k}}^{t}(21) \\
& \left.\mathbf{W}_{\mathrm{k}}^{t}=\mathbf{P}_{\mathrm{k} \mid \mathrm{k}-1}^{t}\left[\mathbf{H}_{\mathrm{k}}^{o}\right]^{t}\right]^{\mathrm{T}}\left(\mathbf{S}_{\mathrm{k}}^{t}\right)^{-1}
\end{aligned}
$$

where $\mathbf{H}_{\mathrm{k}}^{{ }_{t}}$ is the measurement matrix that is calculated for each object pose, $\phi$, and estimated position of track $t$.

\section{Initial Results}

The SBDA track and ID method is evaluated with a Monte Carlo simulation and the performance metric is normalized probability of state error. As detailed in the Figures by the true trajectory, the targets 1) start with position and velocity, 2) pass by each other at a close distance, and 3) finish with a specified direction. Even with a coarse starting position, the tracker was able to locate, track, and identify the targets with clutter.

Figure 5 shows the effectiveness of tracking targets using the JPDAF when targets are separated by a large distance. The separation allows for the determination of a validation gate size that associates the correct measurements to tracks.

Figure 6 shows one case of many where the tracker gets

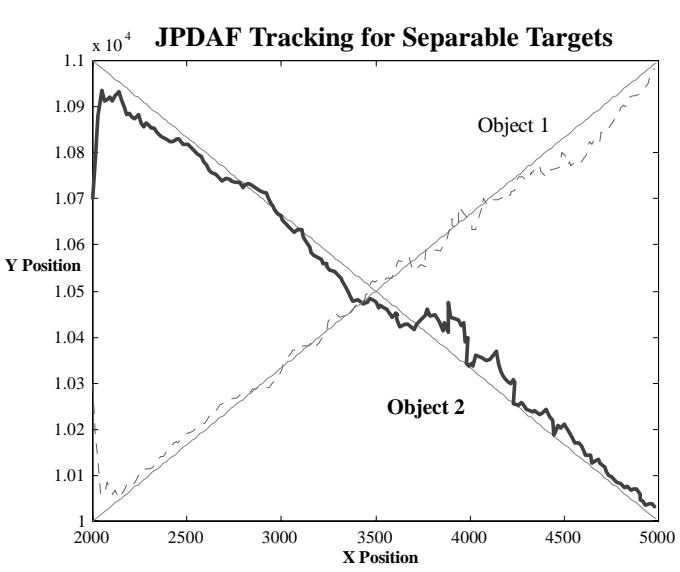

Figure 5. Tracking without ID using JPDAF for targets that are separated.

confused with closely spaced moving targets by the addition of a third target.

Figure 7 shows the same case as Figure 6 with identification information that helps the tracker better

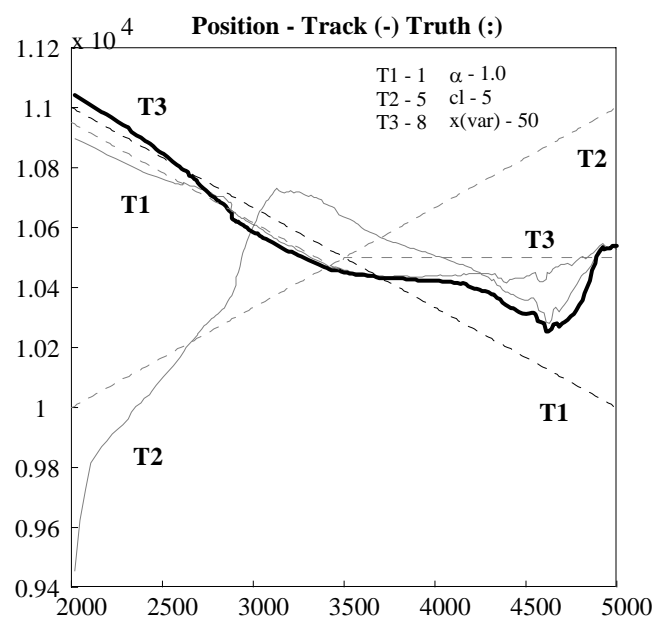

Figure 6. Tracking without ID information.

associate the true position measurements from clutter. In the case presented the clutter comprised of 5 spurious measurements around a target. Target beliefs increased throughout the run as the target was repeatedly identified as the same target.

\section{Discussion \& Conclusions}

Conventional measurement tracking techniques have difficulty with data association when position measurements are close. The SBDA algorithm, which uses the identification information, helps to associate the correct measurement to the correct targets. In the presence of clutter, the novel algorithm utilizes parsimony for processing. The SBDA can be utilized in a timeconstrained weak-sensor scenario to get a general target 
E. Blasgh SBDA Position - Track (-) Truth (:)

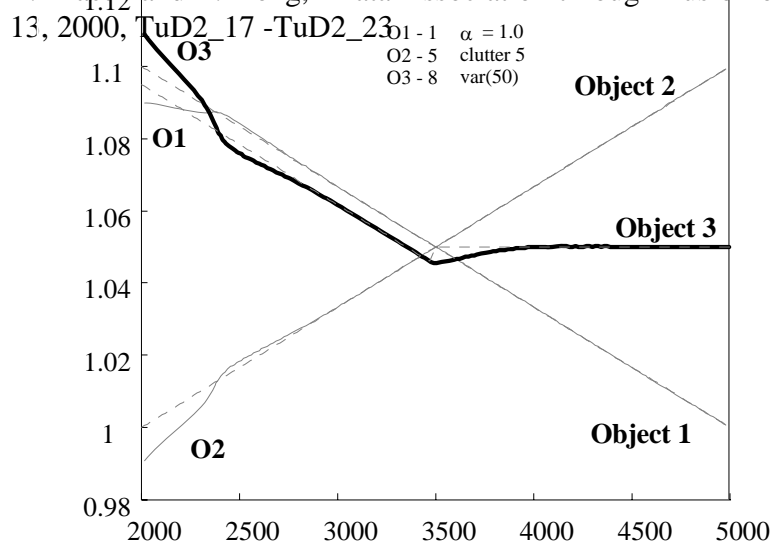

[9] J. Layne, “Automatic Target Recognition and Tracking Filter," SPIE AeroSense - Small Targets, April 1998.

Figure 7. Tracking with ID information.

location and a positive ID due to the fact that the AND function captures positive ID information that can be used to augment a data association tracker.

In a series of simulation experiments, the SBDA performed well resulting in a desirable solution for closely spaced moving targets, and at a faster rate than conventional mutlitarget-multisensor tracking methodologies. The faster rate resulted from a reduction in the gate size to eliminate clutter. The presented technique demonstrates promise for multitarget tracking problems and warrants further exploration in problems where environmental effects, occlusions, lost sensor data, and unknown targets where robust identification information is not available.

\section{References}

[1] Y. Bar-Shalom and X. Li, Multitarget-Multisensor Tracking: Principles and Techniques, YBS, New York, 1995.

[2] X. R. Li and Y. Bar-Shalom, 'Detection threshold selection for tracking performance optimization,' IEEE Transactions on Aerospace and Electronic Systems, Vol. AES-30, No. 3, July 1994, pp. 742-749.

[3] S. Blackman and R. Popoli, Design and Analysis of Modern Tracking Systems, Artech House Publisher, Boston, 1999.

[4] Z. Ding and L. Hong, "Decoupling probabilistic data association algorithm for multiplatform multisensor tracking,” Optical Engineering, ISSN 0091-3286, Vol. 37, No. 2, Feb. 1998.

[5] E. P. Blasch and L. Hong, "Simultaneous Tracking and Identification," Conference on Decision Control, Tampa, FL, December 1998, pg. 249-256

[6] K. Kastella, "Joint multitarget probabilities for detection and tracking," SPIE AeroSense '97, April 2125, 1997.

[7] M. Efe and D. Atherton, “A Tracking Algorithm for both Highly Maneuvering and Non-maneuvering Targets,” CDC '96, San Diego, CA, 1997, pg. $3150-$ 3155.

[8] E. Libby, “Application of sequence comparison methods to multisensor data fusion and target recognition,” Ph.D. Dissertation, AFIT, June 1993. 